Cellular Physiology
and Biochemistry and Biochemistry Published online: September 25, 2015

Accepted: August 12, 2015

This article is licensed under the Creative Commons Attribution-NonCommercial-NoDerivatives 4.0 International License (CC BY-NC-ND) (http://www.karger.com/Services/OpenAccessLicense). Usage and distribution for commercial purposes as well as any distribution of modified material requires written permission.
(C) 2015 The Author(s)

Original Paper

\title{
Impact of Androgen and Dietary Advanced Glycation End Products on Female Rat Liver
}

\author{
Eleni Paliouraa ${ }^{a}$ Sotiria Palimeri ${ }^{b}$ Christina Piperi ${ }^{b}$ Stratigoula Sakellariou ${ }^{c}$ \\ Eleni Kandaraki ${ }^{a}$ Theodoros Sergentanis ${ }^{d}$ Georgia Levidou ${ }^{c}$ George Agrogiannis ${ }^{c}$ \\ Apostolos Papalois $^{\text {e }}$ Penelope Korkolopoulou ${ }^{c}$ Evanthia Diamanti-Kandarakis ${ }^{a}$ \\ Athanasios G. Papavassiliou ${ }^{b}$
}

\begin{abstract}
aThird Department of Internal Medicine, University of Athens Medical School, 'Sotiria' Hospital, Athens, bDepartment of Biological Chemistry, University of Athens Medical School, Athens, 'First Department of Pathology, University of Athens Medical School, Athens, dDepartment of Hygiene, Epidemiology and Medical Statistics, University of Athens Medical School, Athens, experimental-Research Center, ELPEN Pharmaceuticals, Pikermi-Athens, Greece
\end{abstract}

\section{Key Words}

AGEs • Androgen • Liver biochemistry • Immunostaining

\begin{abstract}
Background/Aims: Advanced glycation end products (AGEs) have been related to a wide range of liver disorders including hyperandrogenic states such as the Polycystic Ovary Syndrome (PCOS). The aim of the present study is to evaluate the potential impact of dietary glycotoxins exposure and androgen excess on hepatic histology and biochemistry in an androgenized female rat model. Methods: The study population consisted of 80 female Wistar rats, divided in 3 groups, a group of prepubertal (Group $A, n=30$ ) and adult rats (Group B, n=20) that were androgenized via subcutaneous implantation of dihydrotestosterone-containing pellets as well as a group of adult non-androgenized rodents (Group $C, n=30$ ). All groups were randomly assigned either to a high-AGE or low-AGE diet for 3 months. Results: Rats fed with a high-AGE diet exhibited significantly elevated levels of gamma-glutamyl transferase $(\gamma G T)$ $(p \leq 0.0002)$ and indices of AGE immunostaining in liver tissue $(p<0.01)$ when compared to the respective low-AGE group, while aspartate aminotransferase (AST) levels were affected only in non-androgenized animals $(p=0.0002)$. Androgenization per se constitutes an aggravating factor as demonstrated by the elevated $\gamma$ GT levels in adult androgenized animals compared to non-androgenized, independent of diet content $(p=0.0002)$ and by the elevated AST and alanine aminotransferase (ALT) levels in low-AGE subgroups (adult androgenized vs. nonandrogenized, $p=0.0002$ ) followed by increased immunohistochemical AGE deposition in hepatocytes of the latter categories $(p=0.0007)$. Conclusion: The present study suggests that androgens and glycotoxins may contribute synergistically to distort hepatic physiology and function as observed in hyperandrogenic conditions.

E. Diamanti-Kandarakis and A. G. Papavassiliou contributed equally to this work.

Professor Athanasios G. Papavassiliou

Department of Biological Chemistry, Medical School, University of Athens, 75 M. Asias Street, 11527 Athens, (Greece)

Tel. +30 210746 2508/9, Fax +30 210746 2703, E-Mail papavas@med.uoa.gr
\end{abstract}

KARGER 125 


\section{Cellular Physiology Cell Physiol Biochem 2015;37:1134-1146

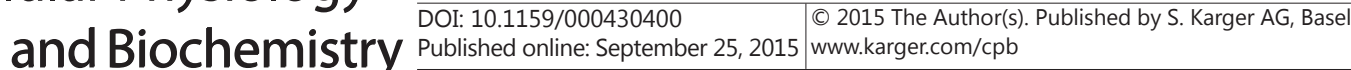 \\ Palioura et al.: Liver Tissue as a Target of Dietary AGEs and Androgen Excess}

\section{Introduction}

Advanced glycation end products (AGEs) constitute a complex and heterogeneous group of compounds derived from the non-enzymatic glycation of proteins, lipids and nucleic acids [1] produced not only endogenously but also from exogenous sources [2]. Westernized diets are considered a fundamental component of exogenously consumed glycotoxins generated under specific cooking methods [2] and are believed to contribute to total body burden of these compounds and to their tissue deposition $[3,4]$.

Crucial to the accumulation and biological action of AGEs is the process of their liver degradation (besides kidney) -and predominantly hepatic sinusoidal endothelial cells and to lesser extent Kupffer cells- being involved in their elimination through a scavenger receptormediated mechanism [5]. Consecutively, any disturbance in liver function could negatively affect hepatic clearance of AGE adducts whereas exposure to increased glycotoxins could adversely impact hepatic sinusoidal elimination and scavenger function [6].

Besides its pivotal role in compound degradation, liver has the potency to generate low levels of reactive oxygen species (ROS) and, in the case of AGEs, is presumed to contribute to inflammatory processes through the AGE-RAGE (Receptor for AGEs) axis expressed in hepatocytes and hepatic stellate cells [7]. In line with this, several human and experimental studies have shown an association between AGEs with a spectrum of liver disorders from simple steatosis and biochemical aberrations to non-alcoholic steatohepatitis and hepatic cirrhosis either as a result of disturbed liver removal [8-10] or as a possible confounding factor to pathophysiology and progression of these diseases through the induction of oxidative stress and inflammation [7]. Regarding exogenous AGEs, experimental evidence confirms their increased serum and liver tissue deposition and also their ability to promote hepatic oxidative stress both in vitro and possibly in vivo affecting liver biochemistry [11-14].

Increased serum AGEs are observed in hyperandrogenic states such as Polycystic Ovary Syndrome (PCOS) [15] in which higher prevalence of abnormal liver biochemistry is also documented $[16,17]$. Although obesity and insulin resistance are the main pathogenetic mechanisms involved, hyperandrogenemia presents an additive adverse factor on liver function of these women [16] though the exact physiological mechanisms are not yet clarified.

Interestingly, in hyperandrogenic states such as PCOS, AGEs are found to be positively correlated with testosterone levels implying an interaction between AGE proteins and hyperandrogenaemia [18]. A previous study from our group has shown the negative impact of androgens and nutritional AGEs in the ovarian tissue of female rats in terms of disturbed detoxification system [19].

Taking all these into consideration, the aim of the present study was to explore whether liver per se could be another possible organ affected by the combined exposure to dietary glycotoxins and androgen excess considering that both parameters may have a negative liver impact.

\section{Materials and Methods}

\section{Animals}

Our study population consisted of 80 female Wistar rats, 30 of which were at the age of 4 weeks (prepubertal stage of life) while the others were at the age of 12-20 weeks (adult life) (Fig. 1). The initial population was divided into 3 main groups: Group A $(n=30)$ was submitted at $4^{\text {th }}$ week of age (prepubertal) to androgenization, a process previously described by Mannerås et al. [20]. Specifically, 90-day continuous release pellets containing $7.5 \mathrm{mg}$ dihydrotestosterone (DHT) were subcutaneously implanted to animals leading to a daily exposure of $83 \mu \mathrm{g}$. This experimental model was selected to mimic both the reproductive and metabolic characteristics of PCOS women, whose DHT levels are found $\sim 1.7$-fold higher than those of healthy women [20]. Group A was subsequently randomly divided into two subgroups based on food content; Subgroup A1 ( $\mathrm{n}=15$ ) was fed commercial chow with low-AGE content (LA) while subgroup A2 (n $=15$ ) was fed commercial chow with high-AGE content (HA).

\section{KARGER}




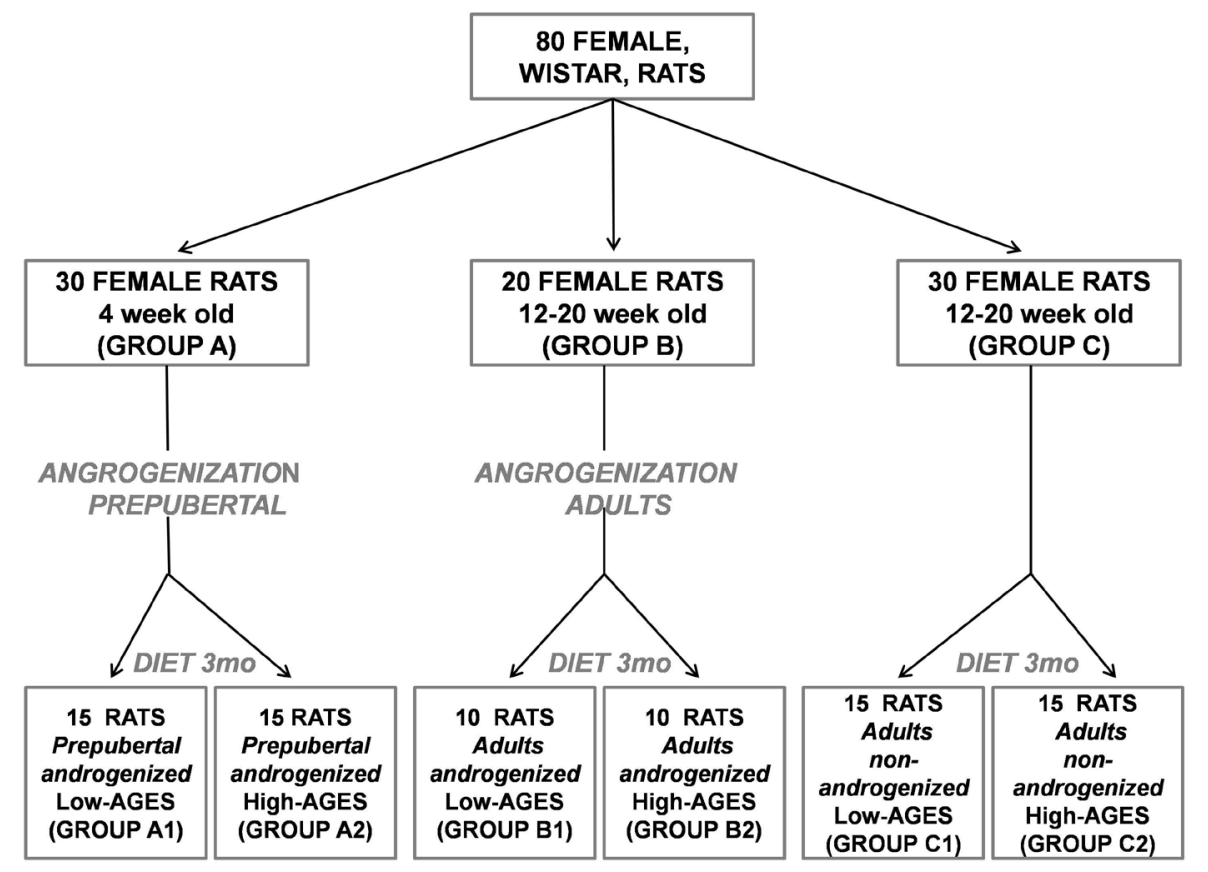

Fig. 1. Schematic representation of the experimental outline and animal allocation into different diet subgroups.

Group B was composed of 20 older rodents, aged 12-20 weeks (adults), which were submitted to the same previously described process of androgenization. Similarly, a further random division into two subgroups based on the criteria of AGE content, followed androgenization. Subgroup B1 $(n=10)$ was fed commercial chow low in AGE content while subgroup B2 $(n=10)$ was exposed to a high-AGE content diet. Finally, group $C$ was composed of 30 non-androgenized animals, which at 12-20 weeks of age, were equally subdivided in two groups. Subgroup C1 $(n=15)$ followed a low-AGE content diet while subgroup C2 $(n=15)$ was fed a high-AGE commercial chow.

The animals were housed four to five per cage under controlled conditions $\left(21-22^{\circ} \mathrm{C}, 55-65 \%\right.$ humidity, 12-h light/12-h dark cycle) and were given pelleted food and water ad libitum at ELPEN (ExperimentalResearch Centre, ELPEN Pharmaceuticals, Athens, Greece). Animal care and experimental procedures conformed to the "Guide for the Care and Use of Laboratory Animals" (Department of Health, Education and Welfare, Athens, Greece) and were approved by the Institutional Animal Care and Use Committee.

The diets used were derived from a single standard rat chow (AIN-93G) purchased from Bioserve (Frenchtown, NJ,USA), consisting of $18 \%$ protein, $58 \%$ carbohydrate, $7.5 \%$ fat and $3.73 \mathrm{kcal} / \mathrm{g}$. Regular AIN93G chow is normally prepared by heating at $190^{\circ} \mathrm{C}$ for $30 \mathrm{~min}$. Analysis of this preparation was performed as previously described [3]. Briefly, it contained $436.9 \pm 88.1 \mathrm{mg} \mathrm{CML} / 100 \mathrm{~g}$ protein, $1.179 .98 \pm 127.90 \mathrm{mg}$ fructoselysine/100 g protein and $302.78 \pm 32.82 \mathrm{mg}$ furosine $/ 100 \mathrm{~g}$ protein and was considered as an HA diet.

The same rodent mix was also prepared without heating. This preparation was of equivalent macroand micronutrient and energy content but contained $7.7 \pm 2.2 \mathrm{mg} \mathrm{CML} / 100$ g protein, $601.01 \pm 17.7 \mathrm{mg}$ fructoselysine $/ 100 \mathrm{~g}$ protein and $154.22 \pm 4.54 \mathrm{mg}$ furosine $/ 100 \mathrm{~g}$ protein and was considered an LA diet.

Body weight was monitored weekly. The study was concluded after 3 months and rats were sacrificed with administration of $20 \mathrm{mg} / \mathrm{mL}$ xylazine hydrochloride and $100 \mathrm{mg} / \mathrm{mL}$ ketamine hydro chloride, under anesthesia with ether, allowing blood sample collection and tissue retrieval.

Hormonal and biochemical assays

Testosterone was measured by enzyme-linked immunosorbent assay (ELISA) using commercially available kits (Calbiotech, CA, USA). Insulin was also quantified using ELISA immunoassays purchased by 


\section{Cellular Physiology Cell Physiol Biochem 2015;37:1134-1146 and Biochemistry Published online: September 25,2015 $\begin{aligned} & \text { (c) } 2015 \text { The Author(s). Published by S. Karger AG, Basel } \\ & \text { www.karger.com/cpb }\end{aligned}$ \\ Palioura et al.: Liver Tissue as a Target of Dietary AGEs and Androgen Excess}

Biovendor Laboratory Medicine (Czech Republic). Serum AGE levels (U/mL) were measured by CML-specific competitive ELISA, as described previously. Serum SGOT, SGPT, $\gamma$ GT, glucose, fructosamine and triglycerides were measured by spectrophotometry using commercially available kits (Bayer Hellas).

\section{Immunohistochemical analysis}

Paraffin-embedded sections of formalin-fixed liver tissue were deparaffinized by xylene and dehydrated in graded ethanol. Sections were treated in 3\% hydrogen peroxide in phosphate-buffered saline (PBS) for $15 \mathrm{~min}$ and then rinsed in PBS. To increase the immunoreactivity of AGEs, the sections were placed in $500 \mathrm{~mL}$ of $0.01 \mathrm{~mol} / \mathrm{L}$ citric acid-buffered solution (pH 7.0) and microwaved at $500 \mathrm{~W}$ for $5 \mathrm{~min}$. After thorough washing, the sections were incubated with normal rabbit serum for $20 \mathrm{~min}$ at room temperature to avoid nonspecific binding of the antibodies.

The sections were then incubated overnight at $4^{\circ} \mathrm{C}$ with the anti-AGE monoclonal antibody 6D12 (0.25 mg/ml stock, dilution 1:50; Research Diagnostics, MA, USA) in PBS containing 1\% bovine serum albumin. Immunoreactivity was detected by the streptavidin-biotin-peroxidase method according to the manufacturer's protocol. The final reaction product was visualized with $3,3^{\prime}$-diaminobenzidine tetrahydrochloride (LSAB detection kit; Dako, CA, USA). Lung tissue sections from diabetic rats were used as positive controls for AGE antibody. Negative controls (for example, liver tissue in which the primary antibody was substituted with non-immune mouse or goat serum) were also stained in each run. The evaluation of the immunostained slides was performed blindly and independently by two pathologists. AGE expression was categorized in four levels according to the staining intensity: 1, very weak; 2, weak; 3, moderate; and 4, strong. AGE expression was examined in intrahepatic biliary cells, sinusoidal cells, Kupffer cells and hepatocytes (nuclear and cytoplasmic staining was evaluated separately for the latter cell type). The different cell types were identified by morphology and location by three experienced pathologists (SS, GL, PK). The extent of AGE expression was assessed as the percentage of positive cells in each cell type out of the total number of cells enumerated. Staining intensity was categorized in four levels: 1 , faint; 2 , weak; 3 , moderate; and 4, strong. An H-score based on the percentage of stained cells multiplied by staining intensity was calculated.

\section{Statistical analysis}

Regarding the examined parameters (body weight at baseline, body weight at 3 months, serum AGEs, AST, ALT, $\gamma$ GT, testosterone, insulin, CRP, serum triglycerides, serum glucose, serum fructosamine, AGEs immunohistochemical score), descriptive statistics were calculated; values are presented as mean \pm standard error (SE).

Given the deviation from normality, as attested by the Shapiro-Wilk test, non-parametric statistical tests were performed. Specifically, the overall heterogeneity between the six groups was evaluated by the Kruskal-Wallis test. Five meaningful pairwise comparisons were a priori constructed in the study design, namely: A1 vs. A2, B1 vs. B2, C1 vs. C2 (to evaluate the high vs. low AGEs comparison), as well as B1 vs. C1, $\mathrm{B} 2$ vs. C2 groups (to address the effects of androgenization per se); all pairwise comparisons were assessed by the Mann-Whitney-Wilcoxon test for independent samples. The level of statistical significance regarding the seven pairwise comparisons was set at $0.05 / 5=0.01$ (Bonferroni correction for multiple comparisons) as appropriate, whereas the level of statistical significance regarding the overall heterogeneity remained at the 0.05 level, given that multiple comparisons did not pertain to the latter overall notion. Statistical analysis was performed with STATA/SE version 13 (Stata Corp., College Station, TX, USA).

\section{Results}

Comparisons pertaining to the effect of AGES

The descriptive statistics regarding body weight and measured serum parameters of the study sample are presented in Table 1. Compared with group A1, group A2 presented with higher AGEs serum levels (as expected, p<0.0001), lower body weight at 3 months (190.7 \pm 7.9 vs. $253.7 \pm 8.8 \mathrm{~g})$, higher $\gamma \mathrm{GT}(15.17 \pm 1.01$ vs. $6.01 \pm 0.31 \mathrm{IU} / \mathrm{L}, \mathrm{p}=0.0001)$, higher serum testosterone (8.96 \pm 0.68 vs. $5.41 \pm 0.49 \mathrm{ng} / \mathrm{mL}, \mathrm{p}=0.0004)$ and higher CRP (437.4 \pm 16.3 vs. $225.5 \pm 10.3 \mu \mathrm{g} / \mathrm{mL}, \mathrm{p}<0.0001)$. Trends towards higher AST $(\mathrm{p}=$ 
$0.024)$, ALT ( $p=0.036)$, serum triglycerides $(p=0.014)$ in group A2 vs. group A1 did not reach formal statistical significance in view of the correction for multiple comparisons (Table 1). Moreover, AGE immunohistochemical $\mathrm{H}$-score was higher in group A2 vs. A1 in sinusoidal ( $\mathrm{p}<0.0001,162$ \pm 78.03 vs. $57 \pm 54.05$; Fig. 2,3 ) and Kupffer cells ( $p=0.0001,169 \pm 66.21$ vs. $53 \pm 43.16$; Fig. 2, 3).

Compared with group B1, group B2 exhibited higher serum levels of AGEs (as expected, $\mathrm{p}=0.0002)$, higher $\gamma \mathrm{GT}(12.79 \pm$ 0.70 vs. $6.59 \pm 0.27 \mathrm{IU} / \mathrm{L}, \mathrm{p}=0.0002$ ) and higher serum testosterone levels (16.27 \pm 2.21 vs. $6.73 \pm 0.44 \mathrm{ng} / \mathrm{mL}, \mathrm{p}=0.0002$ ). Trends towards lower body weight $(\mathrm{p}=$ 0.025 ) in group B2 vs. group B1 did not reach significance given the Bonferroni correction (Table 1). Furthermore, group B2 exhibited higher serum fructosamine levels compared to group B1, with marginal, however, statistical significance $(\mathrm{p}=0.010)$. Interestingly, AGEs H-score was higher in group B2 vs. B1 in all cell types. In detail, B2 cases showed higher AGE H- score in hepatocytes (regarding cytoplasmic staining, $\mathrm{p}=0.0011,87 \pm 35.54$ vs. $35 \pm 21.72$; Fig. 2 , 3 ), intrahepatic biliary cells ( $\mathrm{p}=0.010,272$ \pm 119.19 vs. $96 \pm 67.05$; Fig. 2 , 3), sinusoidal cells ( $p=0.0002,191 \pm 48.68$ vs. $77 \pm 50.15$; Fig. 2, 3) Kupffer cells ( $p=0.0002,259 \pm$ 77.15 vs. $60 \pm 59.43$; Fig. 2,3 ).

Regarding the group C2 vs. C1 comparison, higher AGEs levels (as expected, $\mathrm{p}<0.0001)$, higher AST $(203.4 \pm 12.2$ vs. $131.4 \pm 6.1 \mathrm{IU} / \mathrm{L}, \mathrm{p}=0.0002), \gamma \mathrm{GT}(10.52$ \pm 0.31 vs. $3.67 \pm 0.17 \mathrm{IU} / \mathrm{L}, \mathrm{p}<0.0001)$, testosterone $(9.42 \pm 0.98$ vs. $4.39 \pm 0.63$ $\mathrm{ng} / \mathrm{mL}, \mathrm{p}<0.0001), \mathrm{CRP}(356.3 \pm 11.1$ vs. $176.3 \pm 10.9 \mu \mathrm{g} / \mathrm{mL}, \mathrm{p}<0.0001)$ and serum triglyceride levels $(54.6 \pm 3.0$ vs. $44.6 \pm 1.3$, $\mathrm{p}=0.004$ ) were observed in group C2. Trends towards higher ALT $(p=0.044)$ in group C2 did not reach the Bonferroni-corrected threshold of significance. Once again, AGE immunohistochemical H-score was higher in group C2 vs. C1 in all cell populations and more specifically in hepatocytes (regarding cytoplasmic staining, $\mathrm{p}=0.0006,77 \pm 24.17$ vs. $38 \pm 27.85$ and regarding nuclear staining $p=0.0001,212 \pm 88.51$ vs. $78 \pm 54.68$; Fig. 2 , 3 ), intrahepatic biliary cells ( $\mathrm{p}<0.0001,230$ \pm 76 vs. $53 \pm 44.35$; Fig. 2, 3), sinusoidal cells
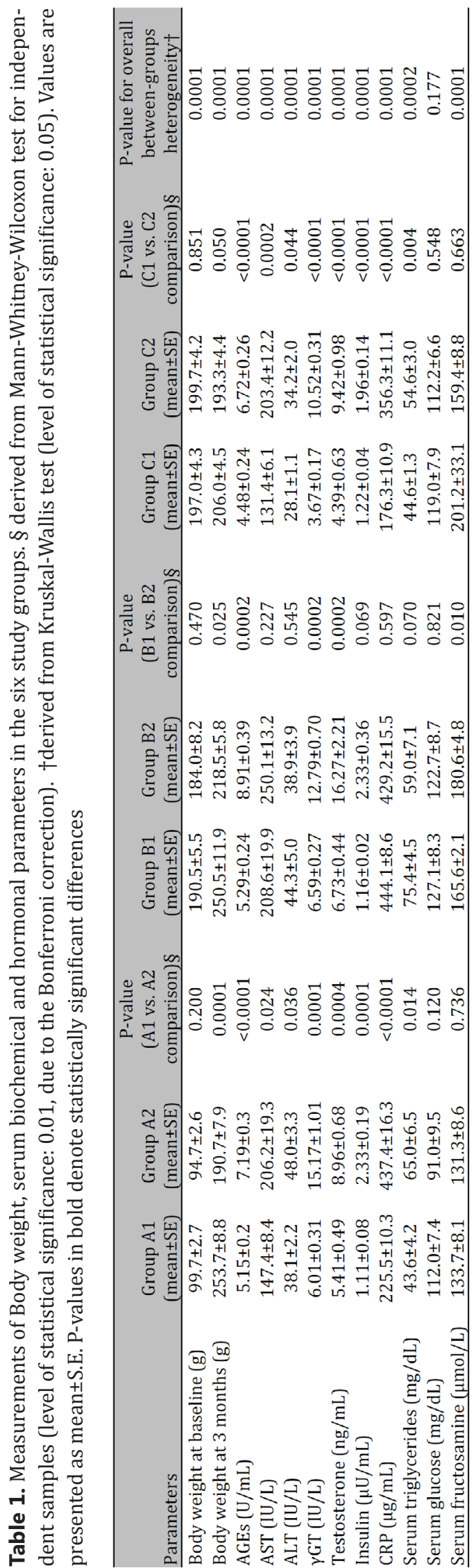


\section{Cellular Physiology Cell Physiol Biochem 2015;37:1134-1146

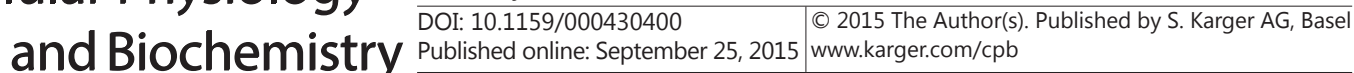
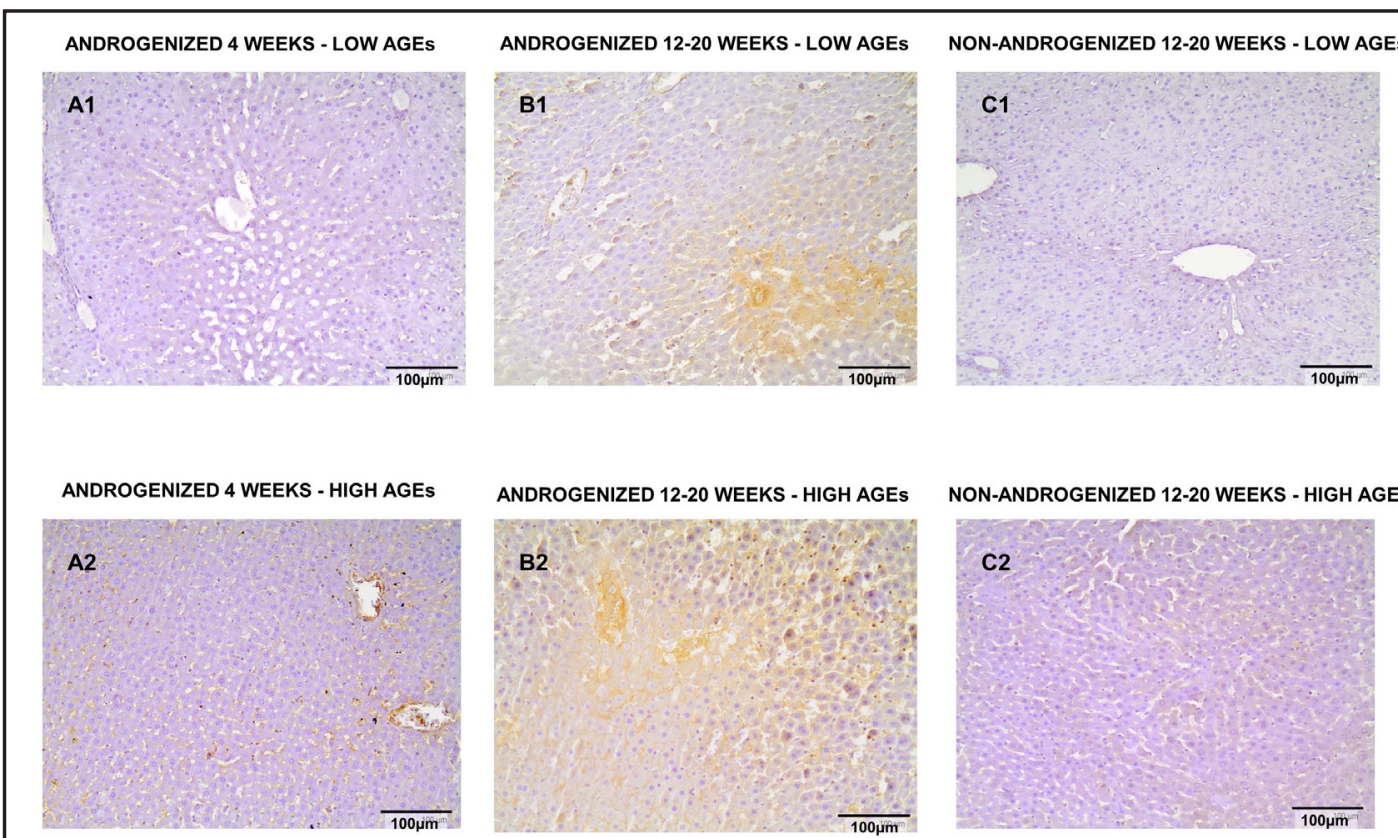

Fig. 2. Immunohistochemical expression of AGEs in different cell types of female rat liver tissue. Higher AGE immunoreactivity is observed in sinusoidal and Kupffer cells of androgenized 4-week-old rats with a high AGE diet (A2) compared to androgenized following a low AGE diet (A1). Adult (12-20 weeks) androgenized rodents following a high-AGE diet (B2) exhibited stronger AGEs immunoreactivity in heatocytes, intrahepatic biliary cells, sinusoidal and Kupffer cells compared to adult androgenized animals following a low AGE diet (B1). Non-androgenized high AGE animals (C2) displayed higher AGE staining in all cell populations compared to non-androgenized low AGE ones (C1). Magnification $\times 200$.

( $\mathrm{p}<0.0001,203 \pm 55.18$ vs. $92 \pm 36.78$; Fig. 2, 3), Kupffer cells ( $<<0.0001,226 \pm 63.91$ vs. 78 \pm 56.45; Fig. 2, 3).

\section{Comparisons pertaining to the effect of androgenization}

Table 2 presents the results of the a priori designed comparisons between the study groups pertaining to the effects of androgenization per se.

Groups C1 and C2 presented with significantly lower weight at three months than groups B1 and B2 ( $p=0.0008$ and $p=0.005$, respectively), although the respective differences were not significant at baseline. Regarding $\gamma$ GT, lower levels were consistently noted in groups $\mathrm{C} 1 / \mathrm{C} 2$ vs. B1/B2 ( $<<0.0001$ and $\mathrm{p}=0.006$, respectively, Fig. $4 \mathrm{~A}$ ); AST and ALT levels were lower in C1 vs. B1 group ( $p=0.002$ for both comparisons, Fig. 4B, C). CRP levels in groups B1/B2 were steadily higher than the respective levels in groups $\mathrm{C} 1 / \mathrm{C} 2$ $(p<0.0001$ and $p=0.002$, Fig. 4D). Group B1 exhibited higher levels of serum triglycerides $(\mathrm{p}<0.0001)$ than group $\mathrm{C} 1$, but these difference was not reproduced at the C2 vs. B2 comparison. Trends towards higher serum fructosamine levels were observed in Group B2 vs. group C2 ( $\mathrm{p}=0.013)$. Finally, AGE immunohistochemical H-score was higher in group $B 1$ vs. C1 in hepatocytes (regarding nuclear staining, $p=0.0007,200 \pm 81.64$ vs. $78 \pm 54.67$; Fig. 3).

\section{Correlation analysis}

At the univariate analysis, serum AST, ALT and $\gamma \mathrm{GT}$ levels correlated positively with AGEs (Spearman's rho $=+0.529, \mathrm{p}<0.0001, \mathrm{rho}=+0.285, \mathrm{p}=0.011$ and $\mathrm{rho}=+0.711, \mathrm{p}<0.0001$, respectively). Similarly, AST, ALT and $\gamma$ GT levels correlated positively with serum testosterone (Spearman's rho $=+0.434, \mathrm{p}=0.0001, \mathrm{rho}=+0.238, \mathrm{p}=0.034$ and $\mathrm{rho}=+0.588, \mathrm{p}<0.0001$, respectively). Serum testosterone correlated positively with AGEs (Spearman's rho $=+0.687$, 


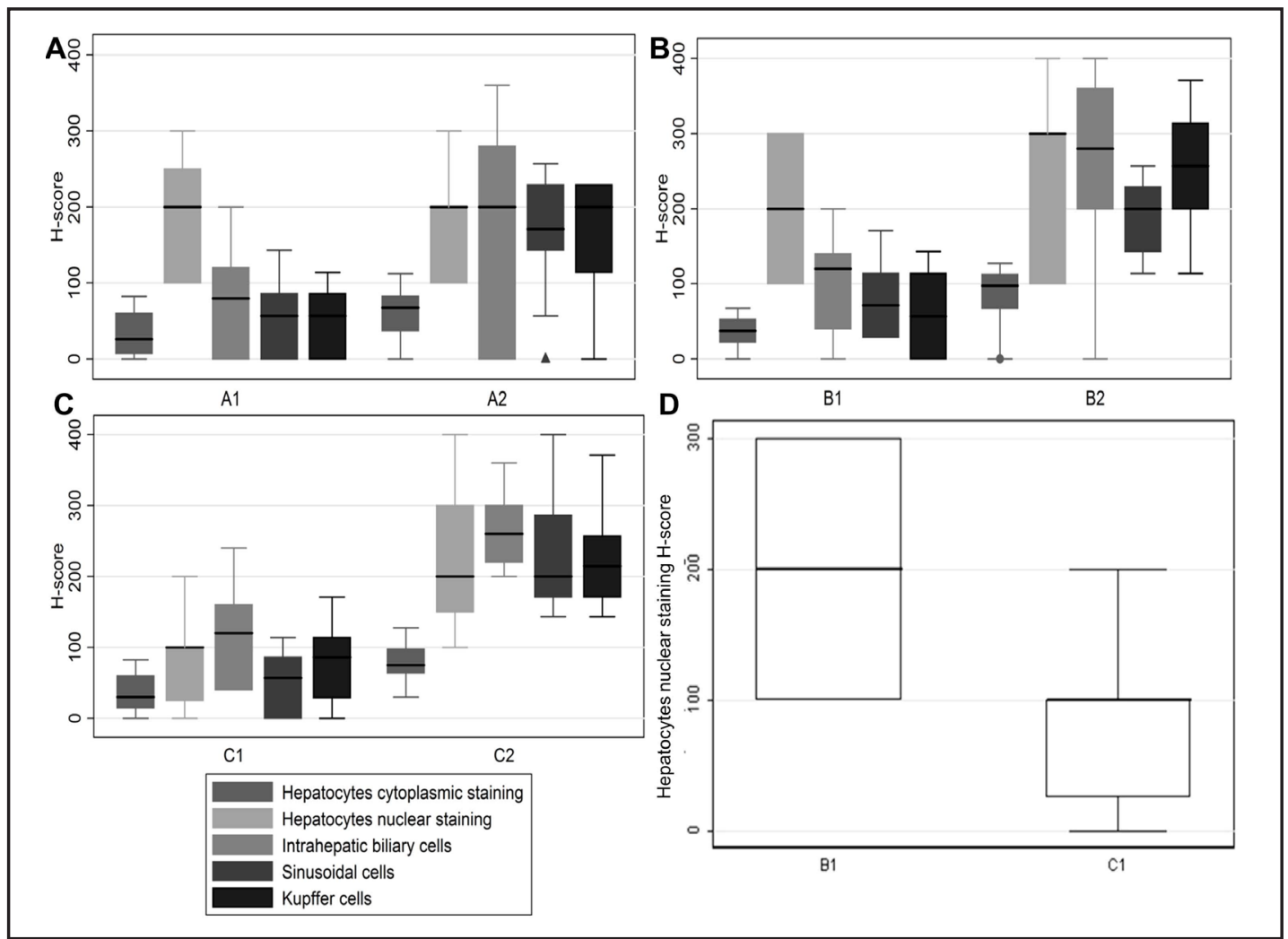

Fig. 3. (A-C) Box plots representing the AGE H-score in all cell populations; hepatocytes (cytoplasmic and nuclear staining), intrahepatic biliary cells, sinusoidal and Kupffer cells. (A) Elevated AGE H-score is observed in all sinusoidal $(\mathrm{p}<0.0001)$ and Kupffer $(\mathrm{p}=0.0001)$ cells of A2 group when compared to A1 group. (B) Elevated AGE H-score is observed in hepatocytes (cytoplasmic staining, $p=0.0011$ ), intrahepatic biliary cells $(\mathrm{p}=0.010)$, sinusoidal $(\mathrm{p}=0.0002)$ and Kupffer $(\mathrm{p}=0.0002)$ cells of B2 group when compared to B1 group. (C) Elevated AGE H-score is observed in hepatocytes (nuclear and cytoplasmic staining, $\mathrm{p}=0.0006$ and $p=0.0001$ respectively), intrahepatic biliary cells $(p<0.0001$, sinusoidal $(p<0.0001)$ and Kupffer cells $(p<0.0001)$ of C2 group when compared to C1 group. (D) Box plots representing the elevated AGE H-score observed in hepatocytes (nuclear staining) of B1 group when compared to C1 group ( $\mathrm{p}=0.0007)$.

$\mathrm{p}<0.0001$ ) and serum $\gamma \mathrm{GT}$ correlated positively with CRP (Spearman's rho $=+0.648$, $\mathrm{p}<0.0001)$.

\section{Discussion}

The main finding of this study relies on the aggravating impact of dietary glycotoxins and androgen excess on liver biochemistry of normal female rats. This was demonstrated by the increased immunohistochemical deposition of glycotoxins in hepatic cell types directly involved in AGE catabolism- such as Kupffer and sinusoidal cells- but also in hepatocytes and intrahepatic biliary cells. The findings are in accordance with the concept that liver is not only an organ of AGE metabolism and degradation [5] but also a site for accumulation of these molecules and probably AGE-related damage [8, 9]. Furthermore, as shown for the first time in the present study, androgen excess has an aggravating impact on several aspects of liver biochemistry parallel to AGE accumulation in hepatocytes of androgenized low-AGE fed animals.

The rats fed with high AGEs for three months showed significantly elevated levels of gamma-glutamyl transferase ( $\gamma \mathrm{GT})$ when compared to the respective low-AGE group. This was 
Table 2. Detailed list of the a priori designed comparisons between the study groups pertaining to the effect of androgenization per se. §derived from Mann-WhitneyWilcoxon test for independent samples (level of statistical significance: $<0.01$ due to the Bonferroni correction). P-values in bold denote statistically significant differences

\begin{tabular}{|c|c|c|}
\hline & \multicolumn{2}{|c|}{ Comparisons pertaining to the effect of androgenization per se } \\
\hline & $\begin{array}{c}\text { P-value } \\
\text { (C:1 vs. B1 comparison)§ }\end{array}$ & $\begin{array}{c}\text { P-value } \\
\text { (6:2 vs. K2 comparison }) \S\end{array}$ \\
\hline \multicolumn{3}{|l|}{ Body weight and serum parameters } \\
\hline Body weight at baseline (g) & 0.344 & 0.051 \\
\hline Body weight at 3 months (g) & 0.0008 & 0.005 \\
\hline AGEs (U/mL) & 0.053 & 0.0005 \\
\hline AST (IU/L) & 0.002 & 0.031 \\
\hline ALT (IU/L) & 0.002 & 0.471 \\
\hline$\gamma \mathrm{GT}(\mathrm{IU} / \mathrm{L})$ & $<0.0001$ & 0.006 \\
\hline $\mathrm{CRP}(\mu \mathrm{g} / \mathrm{mL})$ & $<0.0001$ & 0.002 \\
\hline Serum triglycerides $(\mathrm{mg} / \mathrm{dL})$ & $<0.0001$ & $>0.999$ \\
\hline Scrum glucosc $(\mathrm{mg} / \mathrm{dL})$ & 0.579 & 0.346 \\
\hline Insulin $(\mu \mathrm{U} / \mathrm{mL})$ & 0.577 & 0.503 \\
\hline Serum fructosamine $(\mu \mathrm{mol} / \mathrm{L})$ & 0.120 & 0.013 \\
\hline \multicolumn{3}{|c|}{ Immunohistochemical results - $\Lambda$ GEs immunostaining } \\
\hline Hepalocyles cyloplasmic staining & 0.1969 & 0.3651 \\
\hline Hepatocytes nuclear staining & 0.0007 & 0.7496 \\
\hline Intrahepatic biliary cells & 0.4713 & 0.4840 \\
\hline Sinusoidal cells & 0.2660 & 0.0116 \\
\hline Kupffer cells & 0.3930 & 0.1873 \\
\hline
\end{tabular}

an unchanged finding consistently noted in all under study populations- both androgenized and non-androgenized ones (Fig. 4) - possibly related to the increased glycotoxins deposition in intrahepatic biliary cells. Interestingly, this finding was also observed in previous studies indicating an important role of the biliary system in the disposition of AGE [21]. Furthermore, $\gamma$ GT levels were positively correlated with AGEs implying an aggravating impact of high AGE nutrition on this liver enzyme. According to our knowledge, this is the first study to present a possible association between dietary glycotoxins and $\gamma \mathrm{GT}$ given that this parameter has not been determined in previous studies of rodents fed with diets high in AGEs $[11,13,14]$.

The mechanism via which glycotoxins may exert their negative effect on $\gamma$ GT cannot be clarified from the present study though some assumptions can be proposed. A prominent speculation for elevated $\gamma$ GT levels is that this might reflect a defensive response /adaptation to oxidative stress or inflammatory reactions that allow cells access to increase intracellular glutathione, which is a main protective mechanism against oxidative stress [22]. Indeed, several studies have confirmed the response of $\gamma \mathrm{GT}$ to experimentally-induced oxidative stress in several tissues [22]. As AGEs are potent triggers for ROS production in the liver $[11,12]$, it could be speculated that nutritional exposure to glycotoxins may represent an inflammatory environment, on the basis of elevated serum and hepatic AGEs deposition documented in the present experiment, to stimulate $\gamma$ GT production as defensive mechanism.

Regarding androgen exposure, the current study reveals that androgenized adult animals exhibited significantly elevated $\gamma$ GT levels compared to non-androgenized ones, independent of diet type suggesting that androgens may have an additional unfavorable effect on this enzyme. This finding is in accordance with human studies showing elevated levels of $\gamma$ GT in hyperandrogenic women [16] and furthermore, $\gamma$ GT levels were positively correlated with testosterone implying a possible pathogenetic association between these parameters.

The levels of other liver enzymes- aspartate (AST) and alanine aminotransferase (ALT) - signifying hepatocellular damage are also affected. Specifically, AST levels were found significantly higher in non-androgenized rats fed a high-AGE diet compared to low-AGE ones in accordance with the increased glycotoxins deposition in hepatocytes -both nuclear and cytoplasmic staining- of these animals. This finding agrees with previous studies showing KARGER 


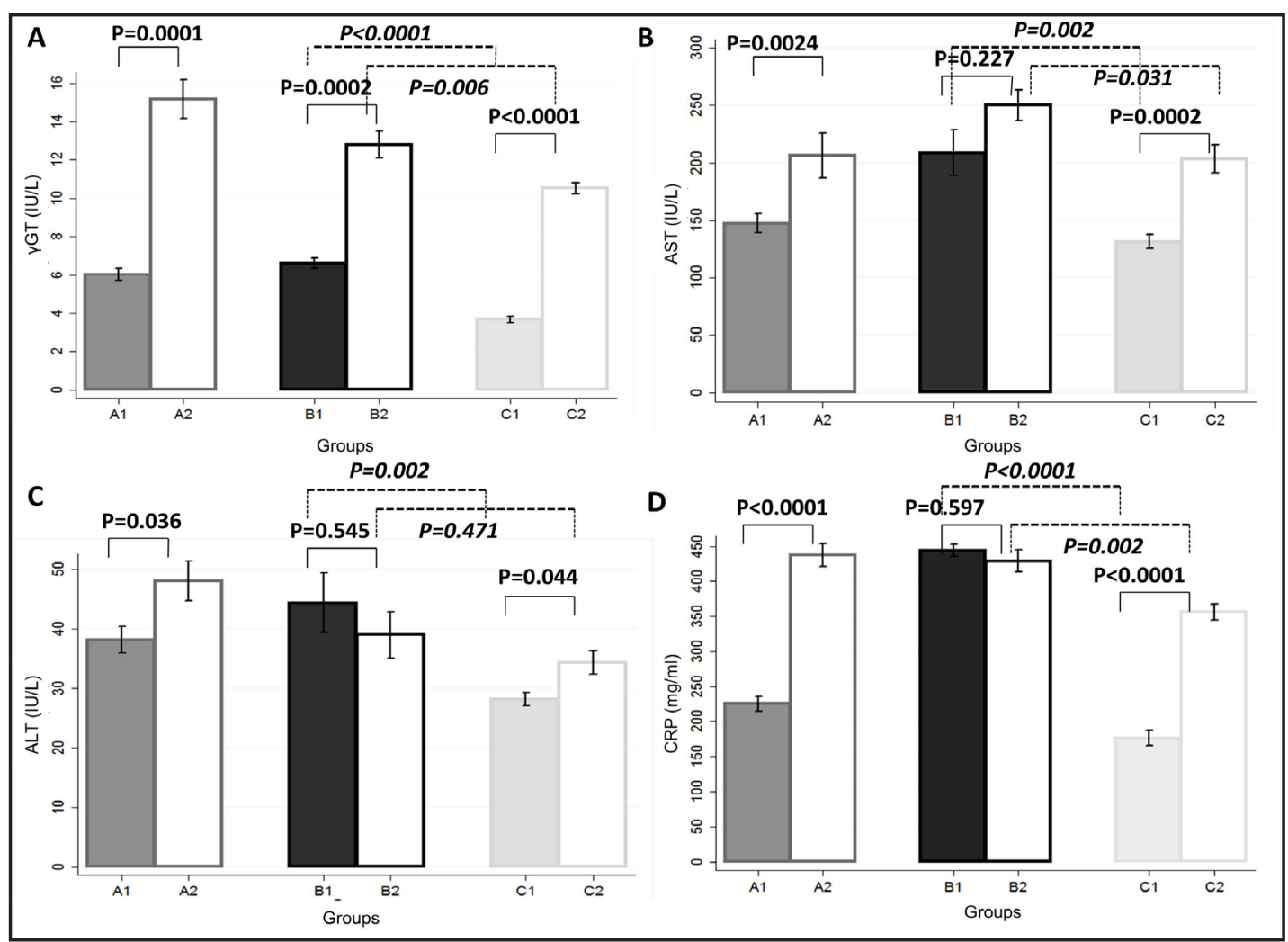

Fig. 4. (A) Effect of high-AGE diet and androgen excess on $\gamma \mathrm{GT}$ levels. Elevated $\gamma \mathrm{GT}$ levels are observed in: (i) all rats following a high-AGE diet (HA) (A2: androgenized prepubertal HA rats, B2: androgenized adults HA rats, C2: non-androgenized HA rats) compared to the respective low-AGE (LA) fed subgroups (A1: androgenized prepubertal LA rats, B1: androgenized adults LA rats, C1: non-androgenized LA rats) and in (ii) androgenized adults rats compared to non-androgenized animals independent of diet (B2: androgenized adults HA rats vs. C2: non-androgenized HA rats and B1: androgenized adults LA rats vs. C1: non-androgenized LA rats). (B) Effect of high-AGE diet and androgen excess on AST levels. Statistically elevated AST levels are observed in: Non-androgenized high-AGE fed animals compared (C2) to non-androgenized low-AGE (C1) and in androgenized adult low-AGE fed rats (B1) compared to the non-androgenized low-AGE fed rats (C1). Trends toward higher AST levels are observed in: androgenized prepubertal high-AGE fed rats (A2) compared to the respective low-AGE group (A1) and in androgenized adult high-AGE fed rats (B2) compared to non-androgenized high-AGE fed rats (C2). (C) Effect of high-AGE diet and androgen excess on ALT levels. Statistically elevated ALT levels are observed in androgenized adult low-AGE fed rats (B1) compared to the non-androgenized low-AGE fed rats (C1). Trends toward higher ALT levels are observed in: androgenized prepubertal high-AGE fed rats (A2) compared to the respective low-AGEs group (A1) and in non-androgenized high-AGE fed rats (C2) compared to respective low-AGE group (C1). (D) Effect of high-AGE diet and androgen excess on CRP levels. Statistically elevated CRP levels are observed in: (i) A2: androgenized prepubertal high-AGE (HA) rats, C2: non-androgenized HA rats compared to the respective low-AGE (LA) fed subgroups (A1: androgenized prepubertal LA rats, C1: non-androgenized LA rats) and (ii) androgenized adult rats compared to non-androgenized animals independent of diet (B2: androgenized adult HA rats vs. C2: non-androgenized HA rats and B1: androgenized adult LA rats vs. C1: non-androgenized LA rats).

increased susceptibility of hepatocytes to the detrimental effects of AGEs from patients with varying degrees of liver impairment $[10,21]$. Furthermore, a trend towards higher AST levels was observed in the group of androgenized prepubertal HA rodents compared to LA ones without reaching statistical significance (Fig. 4B). 


\section{Cellular Physiology Cell Physiol Biochem 2015;37:1134-1146 \\ \begin{tabular}{ll|l} 
DOI: 10.1159/000430400 & O 2015 The Author(s). Published by S. Karger AG, Basel \\
and Biochemistry & Published online: September 25, 2015 www.karger.com/cpb
\end{tabular} \\ Palioura et al.: Liver Tissue as a Target of Dietary AGEs and Androgen Excess}

Concerning the liver enzyme ALT, the impact of high-AGE diet seems to follow the same direction in the above populations though without statistical significance. The androgenized adult rats exhibited no difference for both enzymes which can be attributed to the relatively small number of animals in these subgroups (Fig. 4C).

Elevated ALT and AST levels have been detected in PCOS women $[17,23]$. In this study, the population of androgenized adult animals following a low-AGE, but not a high-AGE diet exhibits significantly higher levels of both aminotransferases when compared with the non-androgenized rats. Interestingly, this is in accordance with the immunohistochemical evidence of increased glycotoxins deposition in hepatocytes (nuclear staining) of androgenized animals shown for the first time in the present study. These findings clearly indicate that androgens per se play an important role in hepatic biochemistry and histology that is more pronounced in the low-AGE diet; nevertheless, in the high-AGE diet androgens do not seem to have an additive effect. This may indicate that the effect of diet on these liver enzymes prevails over the effect of androgenization or it may indicate a compensatory mechanism of action.

Furthermore, the observation of a positive correlation of ALT and AST with androgens seems to be compatible with human studies of hyperandrogenic women showing an association between biochemical and clinical hyperandrogenaemia with elevated ALT levels $[16,17]$. The exact mechanism via which androgen may modify liver biochemistry is yet undetermined. Their role seems to be multifactorial and is believed to be implicated in multiple steps of hepatic physiology. Their impact may be apparent even in intrauterine stages of life by modifying liver metabolism and signaling [24, 25].

The present data of dietary AGEs on liver biochemical profile come in disagreement with recent studies [14]. Patel and colleagues have demonstrated higher levels of aminotransferases in rodents following a regular AGE diet compared to a high AGE one, probably as a consequence of adaptation of liver anti-oxidative mechanism(s) against AGEs. Of note, these measurements were observed at the $39^{\text {th }}$ week of experiment while our findings were on the $12^{\text {th }}$ week when defensive mechanisms could hypothetically be too early to be adapted. It is likely that due to the shorter duration of our study the compensative mechanisms against oxidative and inflammatory status provoked by AGE administration were not yet developed. Furthermore, our study provides evidence that androgen may be a contributing factor to sustain the oxidative stress leading to a further suppress of the antioxidative system.

Another interesting finding is the metabolic phenotype of rats with respect to dietary modification. Elevated fasting insulin levels with normal glucose and fructosamine levels were observed in non-androgenized and androgenized prepubertal HA-fed animals. In adult androgenized rats the impact of dietary AGEs in fasting insulin levels is less prominent, however, the marginal statistical significance of fructosamine levels may imply a distorted metabolic profile. Triglycerides seem to follow the same pattern with a trend towards elevated levels in androgenized prepubertal rats. These metabolic derangements may reflect an indirect impact of diet on liver biochemistry that is independent of the degree of obesity as the high-AGEs diet was related to a lower body weight. Furthermore, given that serum AGEs are known to be associated with insulin resistance [26] this abnormal metabolic profile could imply the development of disturbed insulin action that is known to be pathologically related to hepatic disorders and activation of inflammatory liver pathway [27].

A statistically significant elevation of C-reactive protein (CRP) levels was observed in rats following a high-AGE diet compared to those exposed to a low-AGEs one (apart from androgenized adult group) (Fig. 4D). Furthermore, androgenization per se seems to have an aggravating impact as animals androgenized at adulthood compared to the nonandrogenized control population show higher CRP levels independent of diet. AGEs are proinflammatory molecules shown to indirectly stimulate hepatic CRP production in vitro via stimulation of pro-inflammatory molecules (interleukin-6, interleukin-1 beta) by monocytes that upregulate CRP production in hepatocytes [28]. Furthermore, in an experimental study 


\section{Cellular Physiology Cell Physiol Biochem 2015;37:1134-1146

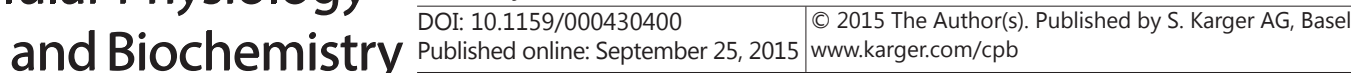 \\ Palioura et al.: Liver Tissue as a Target of Dietary AGEs and Androgen Excess}

of cultured Hep3B cells AGEs were shown to directly upregulate CRP mRNA levels via Rac1 activation [29]. It is likely that their increased serum and liver deposition presented in this study may trigger and further deteriorate a similar biological effect in vivo. A similar pathogenic mechanism may underlie the aggravating impact of androgenization in inflammation as indicated by elevated CRP levels. It is known that in hyperandrogenic states such as in women with PCOS chronic low-grade inflammation is observed as reflected by increased circulating levels of inflammatory mediators [30]. Hyperandrogenemia can induce inflammation in reproductive-age women and one possible mechanism is through the release of cytokines such as tumor necrosis factor alpha (TNF- $\alpha$ ) from mononuclear cells in a receptor-dependent fashion [31], or through enhanced leukocytic reactive oxygen species (ROS) generation [32]. ROS-induced oxidative stress, also triggered by AGEs [33], is a known activator of the nuclear factor-kappaB (NF- $\mathrm{kB})$, a key regulator of inflammation [34]. Activation of this transcription factor presents an intersection point of both androgens and AGEs excess in the induction of inflammatory responses. Therefore, it is speculated that exogenously derived AGEs in combination with increased androgen exposure aggravate local inflammatory processes leading to augmented oxidative stress and release of inflammatory mediators including CRP release from liver.

Interestingly, in this experimental study, the inflammation marker CRP was also positively correlated with $\gamma$ GT. The elevated CRP levels in conjunction with the increased levels of $\gamma$ GT observed in the hyperandrogenic animals compared to the nonandrogenized ones may have implications beyond liver pathology given that these parameters are related to oxidative stress [35].

Another finding of the present study is that all rats fed with high AGEs for three months displayed significantly elevated levels of testosterone when compared to the respective lowAGE group. This was a consistent finding noted in the study populations - both androgenized and non-androgenized animals. This finding is confirmatory of our previous experimental studies presenting a distorted hormonal profile of elevated testosterone levels in normal female rats following a diet rich in glycotoxins [3]. The pathogenic interference between AGEs and androgens is not yet clarified but may be related to the increased distribution of AGEs and RAGE in theca cells of female rats exposed to dietary glycotoxins shown in previous studies [3]. Theca cells are responsible for the androgen production in the ovarian tissue and AGEs may modulate steroidogenesis, therefore a functional link between the increased deposition of AGEs in the ovary and the increased levels of serum testosterone cannot be excluded. Supportive of such interplay is the positive correlation between androgen and serum AGEs levels observed in PCOS women [18]. The differential effects on liver function between endogenous androgens (possibly stimulated by dietary AGEs) and exogenous androgens, is the aromatization of endogenous as opposed to non-aromatizable exogenous androgens. The experimental model of androgenization could indirectly favor the development of a PCOS phenotype exhibiting the hormonal characteristics (hyperandrogenic) of the syndrome. However, the observation of increased androgen levels in non-androgenized animals converges to an additive effect of glycotoxins possibly though stimulation of ovarian androgen production.

This study has some limitations. Although the sample size was satisfactory some differences did not reach statistical significance especially in smaller size subgroups such as the androgenized in adult life population. Additionally, hepatic insulin resistance indexes indicative of the metabolic profile of the exposed animals were not evaluated. Finally, other parameters such as liver organ weight or body weight composition were not estimated.

\section{Conclusion}

The present study shows that high dietary AGEs induce apparent serum and liver tissue accumulation in all experimental groups. This is probably related to disturbed liver biochemistry that is observed throughout the study predominantly by elevation of $\gamma$ GT levels 


\section{Cellular Physiology Cell Physiol Biochem 2015;37:1134-1146 and Biochemistry Published online: September 25,2015 $\begin{aligned} & \text { (c) } 2015 \text { The Author(s). Published by S. Karger AG, Basel } \\ & \text { www.karger.com/cpb }\end{aligned}$ \\ Palioura et al.: Liver Tissue as a Target of Dietary AGEs and Androgen Excess}

compatible with the immunohistochemical evidence of increased deposition in intrahepatic biliary cells. Androgenization per se is shown for the first time to have an additive negative liver impact that is more pronounced for aminotransferases in the low-AGE diet -parallel to the immunohistochemical evidence of increased hepatocyte AGE deposition- and more apparent for $\gamma \mathrm{GT}$ levels, independent of diet.

\section{Disclosure Statement}

The authors declare no financial conflict of interest.

\section{References}

1 Vlassara H, Palace MR: Diabetes and advanced glycation endproducts. J Intern Med 2002;251:87-101.

2 Goldberg T, Cai W, Peppa M, Dardaine V, Baliga BS, Uribarri J, Vlassara H: Advanced glycoxidation end products in commonly consumed foods. J Am Diet Assoc 2004;104:1287-1291.

3 Diamanti-Kandarakis E, Piperi C, Korkolopoulou P Kandaraki E, Levidou G, Papalois A, Patsouris E, Papavassiliou AG: Accumulation of dietary glycotoxins in the reproductive system of normal female rats. J Mol Med (Berl) 2007;85:1413-1420.

4 Koschinsky T, He CJ, Mitsuhashi T, Bucala R, Liu C, Buenting C, Heitmann K, Vlassara H: Orally absorbed reactive glycation products (glycotoxins): an environmental risk factor in diabetic nephropathy. Proc Natl Acad Sci USA 1997;94:6474-6479.

5 Smedsrød B, Melkko J, Araki N, Sano H, Horiuchi S: Advanced glycation end products are eliminated by scavenger-receptor- mediated endocytosis in hepatic sinusoidal Kupffer and endothelial cells. Biochem J 1997;322:567-573.

6 Hansen B, Svistounov D, Olsen R, Nagai R, Horiuchi S, Smedsrød B: Advanced glycation end products impair the scavenger function of rat hepatic sinusoidal endothelial cells. Diabetologia 2002;45:1379-1388.

7 Santos JC, Valentim IB, de Araújo OR, Ataide Tda R, Goulart MO: Development of nonalcoholic hepatopathy: contributions of oxidative stress and advanced glycation end products. Int J Mol Sci 2013;14:19846-19866.

8 Sebeková K, Kupcová V, Schinzel R, Heidland A: Markedly elevated levels of plasma advanced glycation end products in patients with liver cirrhosis - amelioration by liver transplantation. J Hepatol 2002;36:66-71.

9 Yagmur E, Tacke F, Weiss C, Lahme B, Manns MP, Kiefer P, Trautwein C, Gressner AM: Elevation of Nepsilon(carboxymethyl)lysine-modified advanced glycation end products in chronic liver disease is an indicator of liver cirrhosis. Clin Biochem 2006;39:39-45.

10 Hyogo H, Yamagishi S, Iwamoto K, Arihiro K, Takeuchi M, Sato T, Ochi H, Nonaka M, Nabeshima Y, Inoue M, Ishitobi T, Chayama K, Tazuma S: Elevated levels of serum advanced glycation end products in patients with non-alcoholic steatohepatitis. J Gastroenterol Hepatol 2007;22:1112-1119.

11 Cai W, He JC, Zhu L, Chen X, Wallenstein S, Striker GE, Vlassara H: Reduced oxidant stress and extended lifespan in mice exposed to a low glycotoxin diet: association with increased AGER1 expression. Am J Pathol 2007;170:1893-1902.

12 Iwamoto K, Kanno K, Hyogo H, Yamagishi S, Takeuchi M, Tazuma S, Chayama K: Advanced glycation end products enhance the proliferation and activation of hepatic stellate cells. J Gastroenterol 2008;43:298304.

13 Sato T, Wu X, Shimogaito N, Takino J, Yamagishi S, Takeuchi M: Effects of high-AGE beverage on RAGE and VEGF expressions in the liver and kidneys. Eur J Nutr 2009;48:6-11.

14 Patel R, Baker SS, Liu W, Desai S, Alkhouri R, Kozielski R, Mastrandrea L, Sarfraz A, Cai W, Vlassara H, Patel MS, Baker RD, Zhu L: Effect of dietary advanced glycation end products on mouse liver. PLoS One 2012;7:35143.

15 Diamanti-Kandarakis E, Katsikis I, Piperi C, Kandaraki E, Piouka A, Papavassiliou AG, Panidis D: Increased serum advanced glycation end-products is a distinct finding in lean women with polycystic ovary syndrome (PCOS). Clin Endocrinol (Oxf) 2008;69:634-641. 


\section{Cellular Physiology Cell Physiol Biochem 2015;37:1134-1146

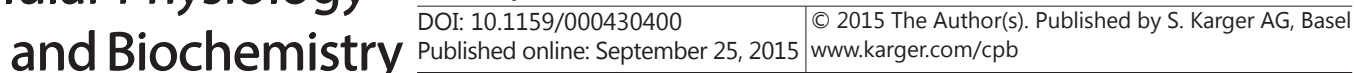 \\ Palioura et al.: Liver Tissue as a Target of Dietary AGEs and Androgen Excess}

16 Economou F, Xyrafis X, Livadas S, Androulakis II, Argyrakopoulou G, Christakou CD, Kandaraki E, Palioura E, Diamanti-Kandarakis E: In overweight/obese but not in normal-weight women, polycystic ovary syndrome is associated with elevated liver enzymes compared to controls. Hormones (Athens) 2009;8:199-206.

17 Schwimmer JB, Khorram 0, Chiu V, Schwimmer WB: Abnormal aminotransferase activity in women with polycystic ovary syndrome. Fertil Steril 2005;83:494-497.

18 Diamanti-Kandarakis E, Piperi C, Kalofoutis A, Creatsas G: Increased levels of serum advanced glycation end-products in women with polycystic ovary syndrome. Clin Endocrinol (Oxf) 2005;62:37-43.

19 Kandaraki E, Chatzigeorgiou A, Piperi C, Palioura E, Palimeri S, Korkolopoulou P, Koutsilieris M, Papavassiliou AG: Reduced ovarian glyoxalase-I activity by dietary glycotoxins and androgen excess: a causative link to polycystic ovarian syndrome. Mol Med 2012;18:1183-1189.

20 Mannerås L, Cajander S, Holmäng A, Seleskovic Z, Lystig T, Lönn M, Stener-Victorin E: A new rat model exhibiting both ovarian and metabolic characteristics of polycystic ovary syndrome. Endocrinology 2007;148:3781-3791.

21 Butscheid M, Hauptvogel P, Fritz P, Klotz U, Alscher DM: Hepatic expression of galectin-3 and receptor for advanced glycation end products in patients with liver disease. J Clin Pathol 2007;60:415-418.

22 Whitfield JB: Gamma glutamyltransferase. Crit Rev Clin Lab Sci 2001;38:263-355.

23 Setji TL, Holland ND, Sanders LL, Pereira KC, Diehl AM, Brown AJ: Nonalcoholic steatohepatitis and nonalcoholic Fatty liver disease in young women with polycystic ovary syndrome. J Clin Endocrinol Metab 2006;91:1741-1747.

24 Hogg K, Wood C, McNeilly AS, Duncan WC: The in utero programming effect of increased maternal androgens and a direct fetal intervention on liver and metabolic function in adult sheep. PLoS One 2011;6:24877.

25 Sun M, Maliqueo M, Benrick A, Johansson J, Shao R, Hou L, Jansson T, Wu X, Stener-Victorin E: Maternal androgen excess reduces placental and fetal weights, increases placental steroidogenesis, and leads to long-term health effects in their female offspring. Am J Physiol Endocrinol Metab 2012;303:1373-1385.

26 Tahara N, Yamagishi S, Matsui T, Takeuchi M, Nitta Y, Kodama N, Mizoguchi M, Imaizumi T: Serum levels of advanced glycation end products (AGEs) are independent correlates of insulin resistance in nondiabetic subjects. Cardiovasc Ther 2012;30:42-48.

27 Gaggini M, Morelli M, Buzzigoli E, DeFronzo RA, Bugianesi E, Gastaldelli A: Non-alcoholic fatty liver disease (NAFLD) and its connection with insulin resistance, dyslipidemia, atherosclerosis and coronary heart disease. Nutrients 2013;5:1544-1560.

28 Li J, Hou F, Guo Z, Shan Y, Zhang X, Liu Z: Advanced glycation end products upregulate C-reactive protein synthesis by human hepatocytes through stimulation of monocyte IL- 6 and IL-1 beta production. Scand J Immunol 2007;66:555-562.

29 Yoshida T, Yamagishi S, Nakamura K, Matsui T, Imaizumi T, Takeuchi M, Ueno T, Sata M: Pigment epitheliumderived factor (PEDF) inhibits advanced glycation end product (AGE)-induced C-reactive protein expression in hepatoma cells by suppressing Rac-1 activation. FEBS Lett 2006;580:2788-2796.

30 Diamanti-Kandarakis E, Paterakis T, Kandarakis HA: Indices of low-grade inflammation in polycystic ovary syndrome. Ann N Y Acad Sci 2006;1092:175-186.

31 González F, Sia CL, Bearson DM, Blair HE: Hyperandrogenism induces a proinflammatory TNF $\alpha$ response to glucose ingestion in a receptor-dependent fashion. J Clin Endocrinol Metab 2014;99:848-854.

32 González F, Nair KS, Daniels JK, Basal E, Schimke JM, Blair HE: Hyperandrogenism sensitizes leukocytes to hyperglycemia to promote oxidative stress in lean reproductive-age women. J Clin Endocrinol Metab 2012;97:2836-2843.

33 Luevano-Contreras C1, Chapman-Novakofski K: Dietary advanced glycation end products and aging. Nutrients 2010;2:1247-1265.

34 Tornatore L, Thotakura AK, Bennett J, Moretti M, Franzoso G: The nuclear factor kappa B signaling pathway: integrating metabolism with inflammation. Trends Cell Biol 2012;22:557-566.

35 Lee DH, Blomhoff R, Jacobs DR Jr: Is serum gamma glutamyltransferase a marker of oxidative stress? Free Radic Res 2004;38:535-539. 